\title{
THE MEAN COUPLING TIME FOR INDEPENDENT DISCRETE RENEWAL PROCESSES
}

UDC 519.21

\author{
M. V. KARTASHOV AND V. V. GOLOMOZYI
}

\begin{abstract}
We consider two independent renewal processes with discrete and, in general, nonidentical distributions of interarrival times. The mean coupling time is estimated via the first two moments of these distributions.
\end{abstract}

\section{INTRODUCTION}

The coupling method (for renewal processes, in particular) is described in [1].

In the current paper, we establish explicit bounds for the mean coupling time in terms of the first two moments of interarrival times in the case of two discrete renewal processes and aperiodic distributions. These estimates are based on explicit inequalities for the minimum of a renewal sequence [2]. The estimates can be applied to prove the ergodicity and stability of Markov processes.

\section{Renewal processes and a Bound For the COUpling time}

Consider two independent sequences $\left(\theta_{l j}, j \geq 1\right), l=1,2$, such that the random variables in each sequence are mutually independent, identically distributed, and assume positive integer values. Let random variables $\tau_{l 0}, l=1,2$, be independent of sequences $\left(\theta_{l j}, j \geq 1\right), l=1,2$, and assume integer nonnegative values. For $l=1,2$, let

$$
\begin{gathered}
g_{l j}=\mathrm{P}\left(\theta_{l 1}=j\right), \quad j \geq 1, \quad \mu_{l}=\mathrm{E} \theta_{l 1}, \quad \mu_{l 2}=\mathrm{E}\left(\theta_{l 1}\right)^{2}, \\
u_{l j}=\sum_{n \geq 0}\left(g_{l}\right)_{j}^{* n}, \quad j \geq 0, l=1,2,
\end{gathered}
$$

be the corresponding distributions, moments, and renewal sequences.

Here and in what follows $a^{* n}$ denotes the $n$-th convolution of a sequence $a=\left(a_{i}, i \geq 0\right)$ with itself. The convolution of two sequences $a$ and $b$ is defined as the sequence

$$
(a * b)_{i}=\sum_{s=0}^{i} a_{i-s} b_{s}, \quad i \geq 0 .
$$

Next we introduce the renewal processes

$$
\tau_{l k}=\tau_{l 0}+\sum_{j=1}^{k} \theta_{l j}, \quad k \geq 0, l=1,2,
$$

and Markov overshoot processes for $t \geq 0, l=1,2$ :

$$
\gamma_{l t}=\inf \left(\tau_{l k}-t, \tau_{l k} \geq t, k \geq 0\right) .
$$

2010 Mathematics Subject Classification. Primary 60J45; Secondary 60A05, 60K05.

Key words and phrases. Renewal theory, renewal sequences, coupling method, coupling time. 
Note that $\gamma_{l 0}=\tau_{l 0}$. The conditional expectation given the initial values is denoted by

$$
\mathrm{E}_{i j} \xi=\mathrm{E}\left(\xi \mid \gamma_{10}=i, \gamma_{20}=j\right)=\mathrm{E}\left(\xi \mid \tau_{10}=i, \tau_{20}=j\right) .
$$

We study the coupling time

$$
\zeta \equiv \inf \left(t \geq 1: \gamma_{1 t}=\gamma_{2 t}=0\right)=\inf \left(\tau_{1 n}: \exists n, k \geq 0: \tau_{1 n}=\tau_{2 k}>0\right)
$$

In the proof below we apply a representation for a coupled renewal process given in [1].

Recall that a discrete distribution is called aperiodic if its support does not belong to any strong lattice in $\mathbb{Z}_{+}$. In the aperiodic case, the renewal processes visit every state in $\mathbb{N}$ with positive probability, that is, the coupling time $\zeta$ is finite almost surely.

Theorem 1. Let the distributions $\left(g_{1 j}\right)$ and $\left(g_{2 j}\right)$ be aperiodic. Assume that $\mu_{l}<\infty$ for $l=1,2$. Then

$$
\mathrm{E}_{00} \zeta=\mu_{1} \mu_{2}
$$

Corollary 1. For all $k \geq 1$,

$$
\begin{aligned}
& \mathrm{E}_{k 0} \zeta \leq \frac{\mu_{1} \mu_{2}-\mathrm{E} \min \left(\theta_{11}, \theta_{21}\right)}{\mathrm{P}\left(\theta_{11}-\theta_{21}=k\right)}, \\
& \mathrm{E}_{0 k} \zeta \leq \frac{\mu_{1} \mu_{2}-\mathrm{E} \min \left(\theta_{11}, \theta_{21}\right)}{\mathrm{P}\left(\theta_{21}-\theta_{11}=k\right)},
\end{aligned}
$$

provided the denominators in (8) are positive.

A more general result uses an extra assumption of the aperiodicity type.

Theorem 2. Assume that $g_{11}+g_{21}>0$ and $\mu_{l 2}<\infty$ for $l=1,2$. Then

$$
\mathrm{E}_{i j} \zeta \leq \max (i, j)+C_{12} 1_{i \neq j},
$$

$$
\begin{gathered}
C_{12}=\left(\mu_{12} / \mu_{1}+\mu_{22} / \mu_{2}-2\right)\left(1-\left(1-\bar{u}_{1}\right)\left(1-\bar{u}_{2}\right)\right)^{-1}, \\
\bar{u}_{l} \geq \exp \left(\mu_{l}\left(1-g_{l 1}\right)^{-1} \ln g_{l 1}\right), \quad l=1,2,
\end{gathered}
$$

for all $i, j \geq 0$ such that $(i, j) \neq(0,0)$.

Remark 1 . The number $\bar{u}_{l}$ is equal to

$$
\bar{u}_{l}=\inf \left(u_{l j}, j \geq 1\right) .
$$

The number $\bar{u}_{l}$ is positive if and only if $g_{l 1}>0$.

The following inequality is simpler for applications.

Corollary 2. Let all the assumptions of Theorem 2 hold. Then

$$
\mathrm{E}_{i j} \zeta \leq i+j+C_{12} .
$$

\section{Proofs}

We introduce the notation

$$
m_{i j}=\mathrm{E}_{i j} \zeta, \quad i, j \geq 0 .
$$

Proof of Theorem 1. Following [1], Chapter II, we define the point renewal processes

$$
N_{l k}=\sum_{j \geq 0} 1_{\left\{\tau_{l j}=k\right\}}, \quad k \geq 0, l=1,2,
$$

and the random sequence

$$
N_{k}^{*}=N_{1 k} \cdot N_{2 k}, \quad k \geq 0 .
$$

Since $\tau_{10}=0=\tau_{20}$ in view of $\gamma_{10}=0=\gamma_{20}, N_{10}=N_{20}=1$, whence $N_{0}^{*}=1$. Further, if the event $N_{k}^{*}=1$ occurs, then $N_{1 k}=1=N_{2 k}$ and thus the distribution of $N_{j+k}^{*}$ is 
uniquely defined for $j \geq 0$ and does not depend on $k$. Therefore the sequence $N_{k}^{*}$ is a point renewal process with the regeneration period

$$
\tau^{*}=\inf \left(k \geq 1: N_{k}^{*}=1\right)=\inf \left(k \geq 1: N_{1 k}=1=N_{2 k}\right)=\zeta .
$$

Further

$$
\mathrm{E} N_{k}^{*}=\mathrm{E} N_{1 k} \cdot \mathrm{E} N_{2 k}=u_{1 k} \cdot u_{2 k}
$$

by definition.

According to the aperiodicity, $u_{1 k} \cdot u_{2 k}>0$ starting with some number $k$. Thus (14) implies the aperiodicity of $\tau^{*}$. By the discrete renewal theorem,

$$
\lim _{k \rightarrow \infty} \mathrm{E} N_{k}^{*}=1 / \mathrm{E} \tau^{*}=1 / \mathrm{E}_{00} \zeta
$$

(see [3]).

On the other hand,

$$
\lim _{k \rightarrow \infty} \mathrm{E} N_{k}^{*}=\lim _{k \rightarrow \infty} u_{1 k} \cdot u_{2 k}=\left(1 / \mu_{1}\right)\left(1 / \mu_{2}\right)
$$

in view of (14) and by the discrete renewal theorem.

Now Theorem 1 follows from the latter equality and (15).

Lemma 1. For $i, j \geq 1$ we have

$$
\begin{gathered}
m_{i j}=1+m_{i-1, j-1} 1_{(i, j) \neq(1,1)}, \\
m_{i j}=\min (i, j)+m_{i-j, 0} 1_{i>j}+m_{0, j-i} 1_{j>i} .
\end{gathered}
$$

The proof obviously follows from (5), since the process $\left(\gamma_{l t}\right), l=1,2$, coincides with the unit speed drift to the point 0 on the set $\left\{\gamma_{l t}>0\right\}$. In particular, $\zeta=i$ for $\gamma_{10}=i=\gamma_{20} \geq 1$.

Proof of Corollary 1. Using definitions (5) and (4) and the total probability law for the first jump in (16), we obtain

$$
\begin{aligned}
m_{00} & =1+\sum_{i \geq 1, j \geq 1} g_{1 i} g_{2 j} m_{i-1, j-1} 1_{(i, j) \neq(1,1)}=1+\sum_{i \geq 1, j \geq 1} g_{1 i} g_{2 j}\left(m_{i j}-1\right) \\
& =\sum_{i \geq 1, j \geq 1} g_{1 i} g_{2 j} \min (i, j)+\sum_{i>j \geq 1} g_{1 i} g_{2 j} m_{i-j, 0}+\sum_{j>i \geq 1} g_{1 i} g_{2 j} m_{0, j-i} \\
& =\mathrm{E} \min \left(\theta_{11}, \theta_{21}\right)+\sum_{k \geq 1} m_{k 0} \mathrm{P}\left(\theta_{11}-\theta_{21}=k\right)+\sum_{k \geq 1} m_{0 k} \mathrm{P}\left(\theta_{21}-\theta_{11}=k\right) .
\end{aligned}
$$

Substituting (7) into the preceding equality we prove (8), since the terms are nonnegative in the latter equality.

Lemma 2. For $i, j \geq 1$, we have

$$
\begin{aligned}
& m_{i 0}=\sum_{j=1}^{i-1} g_{2 j}\left(j+m_{i-j, 0}\right)+i g_{2 i}+\sum_{j>i} g_{2 j}\left(i+m_{0, j-i}\right), \\
& m_{0 j}=\sum_{i=1}^{j-1} g_{1 i}\left(i+m_{0, j-i}\right)+j g_{1 j}+\sum_{i>j} g_{1 i}\left(j+m_{i-j, 0}\right) .
\end{aligned}
$$

Proof. Using (16) we obtain by the Markov property that for $i \geq 1$,

$$
\begin{aligned}
m_{i 0} & =\mathrm{E}_{i 0} \zeta=\mathrm{E}_{i 0} \mathrm{E}\left(\zeta \mid \gamma_{21}\right)=\mathrm{E}_{i 0}\left(\mathrm{E}\left(\zeta \mid \theta_{21}-1\right)\right)=\mathrm{E}_{i 0}\left(1+m_{i-1, \theta_{21}-1} 1_{\left(i, \theta_{21}\right) \neq(1,1)}\right) \\
& =\mathrm{E}_{i 0}\left(m_{i, \theta_{21}}\right) \\
& =\mathrm{E}_{i 0}\left(\theta_{21}+m_{i-\theta_{21}, 0}\right) 1_{\left\{\theta_{21}<i\right\}}+i \mathrm{P}\left(\theta_{21}=i\right)+\mathrm{E}_{i 0}\left(i+m_{0, \theta_{21}-i}\right) 1_{\left\{\theta_{21}>i\right\}} .
\end{aligned}
$$


The proof of the second equality is analogous.

Lemma 3. Let $\left(g_{j}, j \geq 1\right)$ be a discrete distribution. If $u_{r}=\sum_{k \geq 0}(g)_{r}^{* k}$ is the corresponding renewal sequence and $G_{n}=\sum_{j>n} g_{j}$, then

$$
(u * G)_{k} \equiv \sum_{j=0}^{k} u_{k-j} G_{j}=1, \quad k \geq 0 .
$$

The proof of this result can be found in $[3]$.

Proof of Theorem 2. Introduce the functions

$$
v_{1 i}=m_{i 0}-i, \quad v_{2 j}=m_{0 j}-j, \quad i, j \geq 1 .
$$

Note that $v_{l j} \geq 0, j \geq 1, l=1,2$.

Then we consider the sequences $\left(w_{l i}, i \geq 1\right), l=1,2$, defined by equalities

$$
\begin{array}{ll}
v_{1 i}=w_{1 i}+\sum_{1 \leq j<i} g_{2 j} v_{1, i-j}, & i \geq 1, \\
v_{2 j}=w_{2 j}+\sum_{1 \leq i<j} g_{1 i} v_{2, j-i}, \quad j \geq 1 .
\end{array}
$$

Substituting (19) into the definition (20) and taking into account the first equality in (17) we obtain

$$
\begin{aligned}
w_{1 i} & \equiv v_{1 i}-\sum_{1 \leq j<i} g_{2 j} v_{1, i-j}=m_{i 0}-i-\sum_{1 \leq j<i} g_{2 j}\left(m_{i-j, 0}-i+j\right) \\
& =m_{i 0}-\sum_{1 \leq j<i} g_{2 j}\left(j+m_{i-j, 0}\right)-i+\sum_{1 \leq j<i} g_{2 j} i \\
& =i g_{2 i}+\sum_{j>i} g_{2 j}\left(i+m_{0, j-i}\right)-i+\sum_{1 \leq j<i} g_{2 j} i \\
& =i g_{2 i}+\sum_{j>i} g_{2 j}\left(i+v_{2, j-i}-i+j\right)-i+\sum_{1 \leq j<i} g_{2 j} i \\
& =\sum_{j>i}(j-i) g_{2 j}+\sum_{j>i} g_{2 j} v_{2, j-i}, \quad i \geq 1, \\
& w_{2 j}=\sum_{i>j}(i-j) g_{1 i}+\sum_{i>j} g_{1 i} v_{1, i-j}, \quad j \geq 1 .
\end{aligned}
$$

Setting $v_{10}=0$ and $w_{10}=0$, the first equality in (20) becomes the discrete renewal equation for an unknown locally bounded sequence $\left(v_{1 i}\right)$ with locally bounded sequence $\left(w_{1 i}\right)$ on the right hand side. Thus a unique solution of this equation can be represented as the convolution with the renewal sequence $u_{2 j}$ defined by (2):

$$
v_{1 i}=\sum_{s=1}^{i} u_{2, i-s} w_{1 s}, \quad i \geq 0
$$

(see [3]). The index of summation here runs through integer numbers starting with $s=1$ in contrast to [3] and (3), since $w_{10}=0$. 
Consider the sequences

$$
\begin{aligned}
& G_{l s}=\sum_{j \geq 1} g_{l, j+s}=\sum_{j>s} g_{l j}, \quad s \geq 0, l=1,2, \\
& U_{l j}=\left(1 * u_{l}\right)_{j}=\sum_{i=0}^{j} u_{l i}, \quad j \geq 0, l=1,2 .
\end{aligned}
$$

Note that $G_{l 0}=1$.

Substitute the first equality in (21) to (22). Taking into account (23), (24), and Lemma 3 we obtain the equality

$$
v_{1 i}=z_{1 i}+\sum_{j \geq 1} a_{i j}^{(1)} v_{2 j}, \quad i \geq 1
$$

where

$$
a_{i j}^{(1)}=\sum_{s=1}^{i} u_{2, i-s} g_{2, j+s}, \quad i, j \geq 1
$$

$$
\begin{aligned}
z_{1 i} & =\sum_{s=1}^{i} u_{2, i-s} \sum_{j>s}(j-s) g_{2 j}=\sum_{s=1}^{i} u_{2, i-s} \sum_{j>s} g_{2 j} \sum_{r=1}^{j-s} 1=\sum_{s=1}^{i} u_{2, i-s} \sum_{r \geq 1} G_{2, r+s-1} \\
& =\sum_{s=1}^{i} u_{2, i-s}\left(\mu_{2}-\sum_{0 \leq r<s} G_{2 r}\right)=\mu_{2} U_{2, i-1}-\sum_{s=1}^{i} u_{2, i-s}\left(1 * G_{2}\right)_{s-1} \\
& =\mu_{2} U_{2, i-1}-\left(u_{2} * 1 * G_{2}\right)_{i-1}=\mu_{2} U_{2, i-1}-(1 * 1)_{i-1}=\left(\mu_{2} U_{2, i-1}-(i-1)\right)-1 .
\end{aligned}
$$

Now (27) and Daley's inequality [4] imply that

$$
\sup \left(z_{1 i}, i \geq 1\right) \leq \mu_{2} \sup \left(U_{2 i}-i / \mu_{2}, i \geq 0\right)-1 \leq \mu_{2}\left(\mu_{22} / \mu_{2}^{2}\right)-1=\mu_{22} / \mu_{2}-1 .
$$

Further,

$$
\begin{aligned}
\sum_{j \geq 1} a_{i j}^{(1)} & =\sum_{j \geq 1} \sum_{s=1}^{i} u_{2, i-s} g_{2, j+s}=\sum_{s=1}^{i} u_{2, i-s} G_{2 s}=\left(u_{2} * G_{2}\right)_{i}-u_{2 i} G_{20} \\
& =1-u_{2 i} \leq 1-\bar{u}_{2}
\end{aligned}
$$

for $i \geq 1$ in view of equalities (23) and $G_{20}=1$. In (29) we used identity (18) and definition (10).

Similarly, the other equalities in (17), (19), (20), and (21) imply that

$$
v_{2 j}=z_{2 j}+\sum_{i \geq 1} a_{j i}^{(2)} v_{1 i}, \quad j \geq 1
$$

where

$$
\begin{gathered}
z_{2 j}=\mu_{1} U_{1 j}-j-\mu_{1} u_{1 j}=\left(\mu_{1} U_{1, j-1}-(j-1)\right)-1, \\
a_{j i}^{(2)}=\sum_{s=1}^{j} u_{1, j-s} g_{1, i+s}, \quad i, j \geq 1 .
\end{gathered}
$$

Moreover

$$
\begin{gathered}
\sup \left(z_{2 j}, j \geq 1\right) \leq \mu_{1} \sup \left(U_{1 j}-j / \mu_{1}, j \geq 0\right)-1 \leq \mu_{12} / \mu_{1}-1 \\
\sum_{i \geq 1} a_{j i}^{(2)}=1-u_{1 j} \leq 1-\bar{u}_{1} .
\end{gathered}
$$


The condition $g_{11}+g_{21}>0$ implies the aperiodicity of at least one of the renewal processes. The latter inequality proves that $\bar{u}_{1}+\bar{u}_{2}>0$. Substituting (30) into (25) we obtain a linear equation for $\left(v_{1 i}\right)$ with a bounded function on the right hand side (this follows from (28) and (33)). The matrix on the right hand side is the product of matrices $\left(a_{i j}^{(1)}\right)$ and $\left(a_{j i}^{(2)}\right)$. The uniform norm of this matrix does not exceed $\left(1-\bar{u}_{1}\right)\left(1-\bar{u}_{2}\right)<1$ by (29) and (34). Thus the contraction mapping theorem implies that there exists a unique finite nonnegative solution of this equation. Moreover, this solution is bounded and therefore coincides with $\left(v_{1 i}\right)$. This proves that the latter sequence is bounded.

To evaluate the corresponding lim sup in (30) and (25) we apply the inequalities derived above, namely

$$
\begin{gathered}
\sup _{i \geq 1} v_{1 i} \leq \sup _{i \geq 1} z_{1 i}+\left(\sum_{j \geq 1} a_{i j}^{(1)}\right) \sup _{j \geq 1} v_{2 j} \leq \mu_{22} / \mu_{2}-1+\left(1-\bar{u}_{2}\right) \sup _{j \geq 1} v_{2 j}, \\
\sup _{j \geq 1} v_{2 j} \leq \mu_{12} / \mu_{1}-1+\left(1-\bar{u}_{1}\right) \sup _{i \geq 1} v_{1 i} .
\end{gathered}
$$

Substituting (36) into (35) and vice versa and taking into account definitions (19) we prove the first inequality in (9) for both cases $i=0$ and $j=0$ :

$$
\sup _{i \geq 1} v_{1 i} \leq \mu_{22} / \mu_{2}-1+\left(1-\bar{u}_{2}\right)\left(\mu_{12} / \mu_{1}-1+\left(1-\bar{u}_{1}\right) \sup _{i \geq 1} v_{1 i}\right),
$$

whence

$$
\sup _{i \geq 1} v_{1 i} \leq\left(\mu_{22} / \mu_{2}-1+\left(1-\bar{u}_{2}\right)\left(\mu_{12} / \mu_{1}-1\right)\right)\left(1-\left(1-\bar{u}_{1}\right)\left(1-\bar{u}_{2}\right)\right)^{-1} \leq C_{12} .
$$

If $i=j>0$, then inequality (9) becomes an equality in view of (6).

In the general case $i \neq j$, this inequality follows from (16) and (19):

$$
\begin{aligned}
m_{i j} & =\min (i, j)+\left(i-j+v_{1, i-j}\right) 1_{i>j}+\left(j-i+v_{2, j-i}\right) 1_{j>i} \\
& \leq \min (i, j)+\left(i-j+\sup _{i \geq 1} v_{1 i}\right) 1_{i>j}+\left(j-i+\sup _{j \geq 1} v_{2 j}\right) 1_{j>i} \\
& \leq \max (i, j)+C_{12} 1_{i \neq j} .
\end{aligned}
$$

It remains to check the last inequality in (9) to complete the proof of Theorem 2, This inequality follows from (2), (10), and the following two results.

Lemma 4. Let $\left(g_{j}, j \geq 1\right)$ be a discrete distribution and let $u_{n}=\sum_{k \geq 0}(g)_{n}^{* k}$ be the corresponding renewal sequence. Also let

$$
\begin{gathered}
G_{n}=\sum_{i>n} g_{i}, \quad n \geq 0, G_{0}=1, \\
F_{n}=\prod_{s=1}^{n}\left(1-G_{s}\right), \quad 0 \leq n \leq \infty, F_{0}=1,
\end{gathered}
$$

and moreover $0<g_{1}<1$ (that is, $F_{n}>0$ ). Then

$$
u_{n} \geq F_{n} \geq F_{\infty}, \quad n \geq 1 .
$$

The proof follows the lines of [2, Chapter 3.3].

Consider the sequences

$$
\begin{gathered}
f_{n}=F_{n-1} G_{n}, \quad n \geq 1, f_{0}=0, \\
h_{n}=(f * G)_{n}-G_{n}, \quad n \geq 1, h_{0}=0 .
\end{gathered}
$$


Note that

$$
\sum_{s=1}^{n} f_{s}=1-F_{n}, \quad n \geq 1
$$

Indeed, $f_{1}=G_{1}=1-F_{1}$ for $n=1$. By the assumption of induction,

$$
\sum_{s=1}^{n+1} f_{s}=1-F_{n}+F_{n} G_{n+1}=1-F_{n+1} .
$$

Since $G_{n}$ is monotone, (42) implies that

$$
\begin{aligned}
h_{n} & =\sum_{s=1}^{n} f_{s} G_{n-s}-G_{n}=f_{n}+\sum_{s=1}^{n-1} f_{s} G_{n-s}-G_{n} \geq f_{n}+\sum_{s=1}^{n-1} f_{s} G_{n}-G_{n} \\
& =f_{n}+\left(1-F_{n-1}\right) G_{n}-G_{n}=0, \quad n \geq 1,
\end{aligned}
$$

that is, the sequence $\left(h_{n}\right)$ is nonnegative.

By definition (3) of the convolution,

$$
\begin{aligned}
(h * u)_{n} & =\sum_{s=1}^{n} h_{s} u_{n-s}=\sum_{s=1}^{n}\left((f * G)_{s}-G_{s}\right) u_{n-s} \\
& =(f * G * u)_{n}-(f * G)_{0} u_{n}-(G * u)_{n}+G_{0} u_{n}=(f * 1)_{n}-0-1+u_{n} \\
& =1-F_{n}-1+u_{n}=u_{n}-F_{n}
\end{aligned}
$$

in view of (18), (42), and (41).

Now Lemma 4 follows, since the sequence defined by (43) is nonnegative.

Lemma 5. Let $\left(g_{j}, j \geq 1\right)$ be a discrete distribution and let $u_{r}=\sum_{k>0}(g)_{r}^{* k}$ be the corresponding renewal sequence. Put $\mu=\sum_{k \geq 1} k g_{k}$ and $\mu_{2}=\sum_{k \geq 1} k^{2} g_{k}$. If $0<g_{1}<1$, then

$$
\begin{gathered}
\bar{u} \equiv \inf \left(u_{r}, r \geq 1\right) \geq \exp \left(\mu\left(1-g_{1}\right)^{-1} \ln g_{1}\right), \\
\bar{u} \geq \exp \left(\sqrt{\mu_{2}}\left(1-g_{1}\right)^{-1 / 2} \ln g_{1}\right) .
\end{gathered}
$$

Remark 2. One can change the right hand side of (9) by an appropriate modification of inequality (46).

Proof. According to (39), it is sufficient to prove that $F_{\infty}$ is not less than the right hand side of (45) (or the the right hand side of (46)).

The functional $F_{\infty}=F_{\infty}(G)$ here is defined by (38) in terms of the sequence $G=$ $\left(G_{n}, n \geq 1\right)$. If $\mu<\infty$, then the latter sequence belongs to the closed subspace

$$
£_{1}\left(g_{1}, \mu\right)=\left\{G: 0 \leq G_{n+1} \leq G_{n} \leq 1-g_{1}, \sum_{n \geq 1} G_{n} \leq \mu-1\right\} .
$$

Since the functional $F_{\infty}(G)$ is convex on $£_{1}\left(g_{1}, \mu\right)$,

$$
F_{\infty}\left(g_{1}, \mu\right) \equiv \inf _{G \in £_{1}\left(g_{1}, \mu\right)} F_{\infty}(G)
$$

is attained at the boundary of the set $£_{1}\left(g_{1}, \mu\right)$. This set contains the sequences $G$ such that $G_{n} \in\left\{0,1-g_{1}\right\}$ for all $n \geq 1$ except for at most one member. Thus the liminf is attained at the sequence $G^{*}$ such that

$$
G_{k}^{*}= \begin{cases}1-g_{1}, & 1 \leq k<m \\ \mu-1-(m-1) G_{1}^{*} \leq G_{1}^{*}, & k=m \\ 0, & k>m\end{cases}
$$


where $m=\left[\left(\mu-g_{1}\right) /\left(1-g_{1}\right)\right]$. The corresponding minimal value is equal to

$$
\begin{aligned}
F_{\infty}\left(g_{1}, \mu\right) & =\exp \left((m-1) \ln \left(1-G_{1}^{*}\right)\right)\left(1-G_{m}^{*}\right) \\
& \geq \exp \left(m \ln g_{1}\right) \geq \exp \left(\mu\left(1-g_{1}\right)^{-1} \ln g_{1}\right),
\end{aligned}
$$

where we used an obvious inequality $m \leq \mu\left(1-g_{1}\right)^{-1}$.

The proof of (46) is analogous.

\section{BIBLIOGRAPHY}

1. T. Lindvall, Lectures on the Coupling Method, Wiley, New York, 1991. MR,1180522(94c:60002)

2. N. V. Kartashov, Strong Stable Markov Chains, VSP/TViMS, Utrecht/Kiev, The Netherlands/Ukraine, 1996. MR.1451375 (99e:60150)

3. W. Feller, An Introduction to Probability Theory and its Applications, vol. 1, John Wiley \& Sons, New York, 1966. MR0210154 (35:1048)

4. D. J. Daley, Tight bounds for the renewal function of a random walk, Ann. Probab. 8 (1980), no. 3, 615-621. MR573298 (81e:60094)

Department of Probability Theory, Statistics, and Actuarial Mathematics, Faculty for Mechanics and Mathematics, National Taras Shevchenko University, Academician Glushkov Avenue 4E, Kiev 03127, Ukraine

E-mail address: nkartashov@skif.com.ua

Department of Probability Theory, Statistics, and Actuarial Mathematics, Faculty for Mechanics and Mathematics, National Taras Shevchenko University, Academician Glushkov Avenue 4E, Kiev 03127, Ukraine

Received 17/DEC/2010

Translated by O. I. KLESOV 